3) Correction of kinking of proximal vertebral artery by suture fixation 10

4) Correction of kinking of proximal vertebral artery by prothesis of

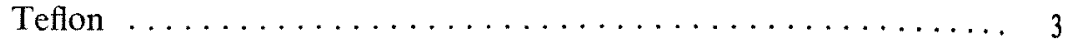

5) Uncal osteophytectomy, unroofing of transverse foraminae and periarterial denervation of cervical vertebral artery ........ 19

6) Powers' operation for syndrome of intermittent vertebral artery

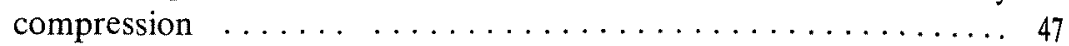

In follow-up study, longest 2 years, gratifying results were obtained. No operative death. The authors emphasized the need for a thorough evaluation of the entire extra and intracranial circulation by arteriography, including the carotid, vertebral, and subclavian artery on both side.

\title{
f-10. Anastomosis of Middle Cerebral Artery with External Carotid Branch for a Treatment of Middle Cerebral Occlusion
}

\section{An Introduction of Microsurgical Technique "Canulation and Coating Method"}

\author{
Toshio Matsubara \\ Neurosurgical Clinic, Okehazama Hospital
}

\begin{abstract}
Anastomosis of middle cerebral artery with external carotid branch was performed as a collateral circuit formation for a treatment of middle cerebral occlusion. The author was able to perform this operation successfully by means of a special technique of vascular microsurgery with an application of tissue adhesive agent as a coating material. This special technique has much simplified the procedure of small vessel anastomosis, with canulation keeping the internal vascular space safely and with no forceps folding the end of vessel wall, thus practically any small vessel can be treated as far as the canulation is possible.

Method: A canule of $1 \mathrm{~mm}$ of caliber is inserted in a parietal branch of superficial temporal artery and the branch is detached from the inside of the temporal skin; the tip of the canule is taken out from the frontal branch of this artery; then, the branches are introduced into the intracranial space, penetrating the temporal muscle and temporal bone hole. A small insicion is made on the vacant middle cerebral branch and another end of the canule which is in the parietal branch of superficial temporal artery is inserted through this incision opening, and the anastomosis was performed by suture and coating of this portion. The canule was removed from the frontal branch and the branch was ligated at the last stage of this operation.

Although the blood flow through this anastomosis may be small and not enough in amount, the first prupose of this operation has been attained as the
\end{abstract}


first step of surgery of this lesion, if the brain is kept off from irreversible ischemic damage.

\title{
f-11. Application of Huge Electrical Magnet for Artificial Embolization
}

\author{
Kenichiro Sugita, Terushige Dor, Yoshiro TaKaoka, Naomi Mutsuga, \\ Ryuichi Tsugane and Osamu Sato \\ Ist Department of Surgery, Nagoya University School of Medicine
}

Since the report of A.J. Luessenhop, the method of artificial embolization has been improved in several aspects, however, it is still problematical how to introduce the emboli to the selected vessels and it is also the matter of debate how to leave or keep the material at the initially introduced sites. We have experienced a case on which introduction of the steel balls into the feeding vessels of a tumor by means of extracranially placed magnetic device was applied.

Magnetic field of the electrical magnet made by Schumann was 7,000 Gauss at the distance of $1 \mathrm{~cm}$ from the tip and 500 Gauss at $10 \mathrm{~cm}$. In the experiments with water flow in the $\mathrm{T}$ or $\mathrm{Y}$ shaped glass tube models, the maximum distance between the bifurcation of glass tube and the tip of magnet was measured at various water pressures, at which the steel ball could be controlled to flow into the aimed direction. The relationship between the distance and the water pressure was as follows; $5 \mathrm{~cm}$ at $150 \mathrm{mmHg}, 7 \mathrm{~cm}$ at $80 \mathrm{mmHg}$ and $9 \mathrm{~cm}$ at $50 \mathrm{mmHg}$. The inner diameter of glass tube $(5-2 \mathrm{~mm})$ an dthe diameter of steel ball $(3.0-$ $0.8 \mathrm{~mm}$ ) had practically no influence on distance-pressure relationship.

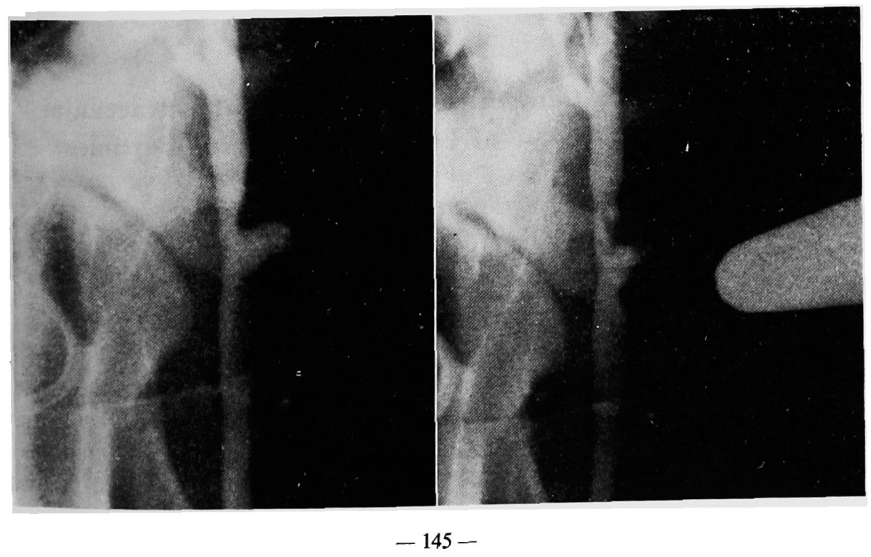

